\title{
A METHOD FOR THE POSITIONING AND ORIENTATION OF RAIL-BOUND VEHICLES IN GNSS-FREE ENVIRONMENTS
}

\author{
R. Hung ${ }^{\text {a,* }}$, B. A. King ${ }^{\text {a }}$, W. Chen ${ }^{\text {a }}$ \\ ${ }^{a}$ Dept. of Land Surveying and Geo-Informatics, The Hong Kong Polytechnic University, Hung Hom, Kowloon, Hong Kong - \\ raymond.hung@connect.polyu.hk, (bruce.king,wu.chen)@ polyu.edu.hk
}

Commission I, ICWG I/Va

KEY WORDS: Rail-bound Navigation, Railway, GNSS-free, Position and Orientation System, Track Alignment Positioning

\begin{abstract}
:
Mobile Mapping System (MMS) are increasingly applied for spatial data collection to support different fields because of their efficiencies and the levels of detail they can provide. The Position and Orientation System (POS), which is conventionally employed for locating and orienting MMS, allows direct georeferencing of spatial data in real-time. Since the performance of a POS depends on both the Inertial Navigation System (INS) and the Global Navigation Satellite System (GNSS), poor GNSS conditions, such as in long tunnels and underground, introduce the necessity for post-processing. In above-ground railways, mobile mapping technology is employed with high performance sensors for finite usage, which has considerable potential for enhancing railway safety and management in real-time. In contrast, underground railways present a challenge for a conventional POS thus alternative configurations are necessary to maintain data accuracy and alleviate the need for post-processing. This paper introduces a method of rail-bound navigation to replace the role of GNSS for railway applications. The proposed method integrates INS and track alignment data for environment-independent navigation and reduces the demand of post-processing. The principle of rail-bound navigation is presented and its performance is verified by an experiment using a consumer-grade Inertial Measurement Unit (IMU) and a smallscale railway model. The method produced a substantial improvement in position and orientation for a poorly initialised system in centimetre positional accuracy. The potential improvements indicated by, and limitations of rail-bound navigation are also considered for further development in existing railway systems.
\end{abstract}

\section{INTRODUCTION}

Mobile Mapping Systems (MMS) are usually employed for outdoor dynamic spatial measurements; their performance have been extensively reviewed for more than a decade (Ellum and El-Sheimy, 2002; El-Sheimy, 2005; Puente et al., 2011; Puente et al., 2013). The majority of current commercial MMS utilise high performance Position and Orientation Systems (POS) for direct georeferencing, which has improved the efficiency of spatial data acquisition compared to those of the previous decade. While the general MMS configuration is theoretically capable of real-time applications, post-processing is usually required to ensure the specified accuracy of the produced point cloud (Gräefe, 2007).

The application of MMS to railways for maintenance and engineering design purposes including measurements of track, power cables, clearance profiles, tunnels and rolling stock, has been reported by several authors including Gräefe (2008) and Leslar et al. (2010). Their application in underground railway environments, where the satellite positioning component is ineffective, has received little attention. This paper therefore presents a potential solution to the positioning of an MMS in underground railways via the integration of an inertial positioning component and railway alignment data.

\subsection{Mobile Mapping in Railways}

Through the integration of Inertial Navigation System (INS) and Global Navigation Satellite System (GNSS), a POS provides reliable performance under good GNSS conditions. GNSS outage is the predominant problem affecting MMS accuracy, especially in long tunnels and underground operations. A number of techniques have been adopted to handle GNSS outage problems such as smoothing algorithms (Nassar et al., 2005), velocity updates/zero velocity updates, landmark updates (Imanishi et al., 2011; Klein and Filin, 2011); and photogrammetric bridging (Hassan et al., 2006). In addition, high performance sensors are utilised to maximise the accuracy for designated railway applications, e.g. RailMapper, Teledyne Optech Lynx MG1, Topcon IP-S2, and Trimble MX8. Nevertheless, the problem of long-term GNSS outage remains a problem for furthering potential applications and developments in underground railways such as those found in Hong Kong and other urban cities.

Alternative railway laser scanning solutions have been concisely reviewed by Hung et al. (2015). Essentially, these systems remove the POS and the data is locally referenced to the track via laser profiles. This indirect spatial referencing introduces restrictions on scanning orientation and limits the potential functions of mobile mapping, including train motion monitoring and train detection.

\subsection{Fusion of Inertial Navigation and Railways}

To overcome the problems of GNSS-free operation in underground railways, the concept of an Underground Railway Laser Scanning (URLS) system has been introduced by Hung et al. (2015). The article addresses several key issues regarding GNSS-free configuration, direct georeferencing, indirect trajectory refinement and potential difficulties. 
Although GNSS may not be totally unavailable for any railway systems, a GNSS-free solution is beneficial for minimizing external environmental impacts and achieving higher consistency of data. In railways, numerous control systems are available for speed restriction, train detection and localisation, which are potential alternatives for the GNSS replacement.

\subsection{Direct Georeferencing in GNSS-free Railways}

Due to the variety of such control systems, identifying a generic solution is difficult. One component that is ubiquitous to railway systems is that of track alignment. This paper proposes the use of rail-bound navigation to replace the GNSS component of the POS in the URLS configuration. The solution is formulated by integrating the inertial navigation and track alignment data for train navigation and direct georeferencing. An experimental study using a consumer-grade IMU and a model train was conducted to investigate the capability and limitations of the proposed solution. The result illustrates the differences between a pure inertial solution and by incorporating rail-bound navigation into the solution. With the latter showing a promising improvement in position accuracy over the former.

\section{RAIL-BOUND NAVIGATION}

Rail-bound navigation combines both inertial navigation and track alignment data. Although track alignment data itself is capable of acting as a direct GNSS replacement, combining it with INS offers enhanced data processing opportunities and possibilities for greater system optimisation.

\subsection{Track Alignment Positioning (TAP)}

The rail track is a fundamental and continuous feature in all railway systems. Track alignment generally comprises two parts: horizontal (straights, tangent points, and circular and spiral curves), and vertical (grades and vertical curves). Alignments are referenced to the rail's centreline in terms of chainage, the horizontal distance along the track's centreline from a defined origin.

2.1.1 Track-level frame: The horizontal and vertical track alignments are defined in a track-level frame $(x-y-z)$, while the absolute position and orientation of the this frame are expressed in a grid coordinate frame (X-Y-Z). The origin of the track-level frame is referenced to the centre of alignment at a given chainage, while the $\mathrm{x}, \mathrm{y}$ and $\mathrm{z}$ axes are defined as the forward, rightward and downward directions respectively along the horizontal alignment. Consequently, the horizontal alignment is defined in the $x-y$ plane, and the vertical alignment is defined in but opposite to the $\mathrm{z}$ axis direction.

2.1.2 Horizontal alignment: Horizontal track alignment can be generalised into a function of chainage for the computation of position, followed by a two-dimensional transformation with respect to the origin:

$$
r_{i}^{n}=r_{0}^{n}+C_{t}^{n} \Delta r_{i}^{t}
$$

where $r_{i}{ }^{n}=\left[\begin{array}{lll}X_{i} & Y_{i} & \psi_{i}\end{array}\right]^{T}$ are the horizontal grid coordinates and the tangent heading at chainage $i$ respectively; $r_{0}{ }^{n}=\left[\begin{array}{lll}X_{0} & Y_{0} & \psi_{0}\end{array}\right]^{T}$ are the horizontal grid coordinates and heading at the origin respectively; $C_{t}^{n}$ is the rotation from the horizontal track-level frame at the origin; and $\Delta r_{i}^{t}=\left[\begin{array}{lll}x_{i} & y_{i} & \Delta \psi_{i}\end{array}\right]^{T}$ are related to the alignment geometry in track-level frame at $i$.

Cant (cross-track tilt) is employed in curve sections to enhance safety at a designated train speed and is expressed as a height difference between the left and right rails. Constant cant is used for circular curves, while zero cant is assigned to non-curve sections or sections with very large radii of curvatures. Cant is introduced to or removed from an alignment linearly via a spiral curve which is used to link various straight and circular alignment components.

2.1.3 Vertical alignment: The vertical track alignment can be approximated by a parabolic function, while the reduced level and grade at a specific chainage can be obtained through Equation (2).

$$
\left[\begin{array}{c}
h_{i} \\
g_{i}
\end{array}\right]=\left[\begin{array}{c}
a_{0}+a_{1} L_{i}+a_{2} L_{i}^{2} \\
a_{1}+2 a_{2} L_{i}
\end{array}\right]
$$

where $h_{i}$ and $g_{i}$ are the reduced level and grade at $i$ respectively; $a_{0}, a_{1}$ and $a_{2}$ are the parabolic coefficients of vertical curve; $L_{i}$ is the chainage difference between the origin and $i$.

2.1.4 Position and orientation: The nominal position of a point along the alignment $\left(X_{i}, Y_{i}, H_{i}\right)$ is capable of being a direct replacement for GNSS in a loosely coupled POS configuration. In addition, the roll and pitch of the track with respect to the levelled frame can also be estimated from the cant and grade as shown in Equation (3).

$$
\left[\begin{array}{c}
\phi_{i} \\
\theta_{i}
\end{array}\right]=\left[\begin{array}{l}
\sin ^{-1} \frac{c_{i}}{w} \\
\tan ^{-1} g_{i}
\end{array}\right]
$$

where $\phi_{i}, \theta_{i}$ are the roll and pitch angles respectively; $c_{i}$ is the cant at $i$; and $w$ is the width of track gauge.

Figure 1 illustrates a flow chart for TAP with the corresponding input, process and output components. Three-dimensional position and orientation of the track centre can be estimated to support the nominal train navigation through the speed integration or alternative chainage data.

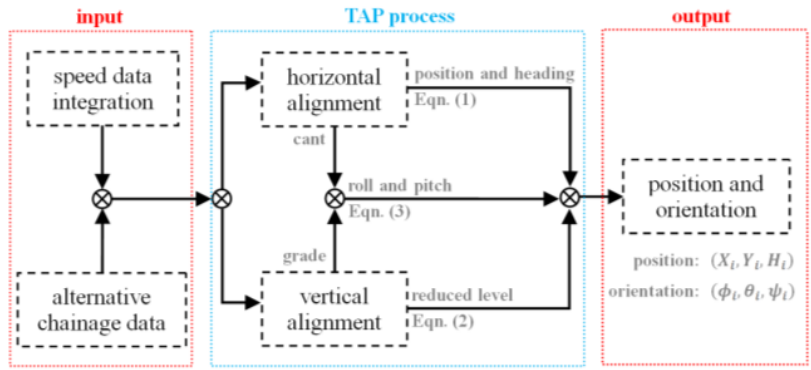

Figure 1. Flow chart for Track Alignment Positioning. 
The physical conditions of rail track are not perfectly described by track data because of construction tolerance, wear and tear, and maintenance work. The resulting position and orientation are also not capable of recovering the dynamic motion of a train; however, it is probably sufficient to serve as a nominal navigation reference by integrating with inertial navigation.

\subsection{Inertial Navigation}

Inertial navigation utilises one or multiple Inertial Measurement Units (IMU) to sense the inertial motion of the mounted body. Body motion is quantified by position and orientation through the integration of sensed accelerations and angular velocities via the INS mechanisation equations (Roger, 2000; Farrell, 2008). The long-term accuracy of inertial navigation depends on several factors, which are summarised in the following sections.

2.2.1 Sensor bias estimation: Although an IMU is usually pre-calibrated before use, the stability of sensor bias is a concern for the performance of inertial navigation, especially during GNSS-outage. Time-correlated sensor bias must be estimated and compensated in order to achieve long-term accuracy. For rate gyroscope, the bias can be estimated through the average output during static initialisation (Woodman, 2007).

2.2.2 System initialisation: Since an INS only maintains the navigation trajectory by tracking the body's inertial motion, initial position, velocity and orientation are required for defining the body reference frame. Initialisation and alignment are usually implemented via integration of stationary or dynamic approaches with external sources, such as GNSS and manual input.

Theoretically, the inertial measurements indicate the orientation of the body frame with respect to the global frame through the alignment process (Grewal et al., 2007; Farrell, 2008). However, this may not be practicable because of the magnitude of sensor errors, especially for low-grade Micro-ElectroMechanical Systems (MEMS) sensors. Despite such errors, the sensed gravity enables coarse alignment for roll and pitch estimation as shown in Equation (4) (Farrell, 2008), while the heading is aligned with the track estimated orientation.

$$
\left[\begin{array}{l}
\phi \\
\theta
\end{array}\right]=\left[\begin{array}{c}
\tan ^{-1}\left(a_{y} / a_{z}\right) \\
\tan ^{-1}\left(-a_{x} / \sqrt{a_{y}^{2}+a_{z}^{2}}\right)
\end{array}\right]
$$

where $a_{x}, a_{y}, a_{z}$ are the normalised accelerometer outputs in the $x, y$, and $z$ body frame coordinate axes.

2.2.3 Navigation error controls: After system initialisation, inertial navigation is supported by integration of compensated inertial measurements, which are usually expressed in the locallevel frame for land-based applications. Although traditional INS mechanisation is rigorous and provides performance with high frequency and precision, error accumulation is inevitable for dead-reckoning applications.

To achieve desirable performance, a system requires accurate alignment for gravity compensation from the sensed accelerometer outputs. The orientation accuracy, however, is degraded by inappropriate alignment and integrated gyroscopic errors, which has contributed greater impacts on position and velocity accuracy than accelerometer errors do (Chow, 2011).
For a general POS configuration, GNSS is widely employed to constrain the growth of navigation errors, while several system parameters are indirectly estimated from GNSS measurements. To improve the long-term accuracy, rigorous dynamic and stochastic models have to be thoughtfully defined for INS/GNSS coupling through appropriate filtering techniques, e.g. Extended Kalman Filter (EKF), Unscented Kalman Filter (UKF) or Particle Filter (PF). Alternative approaches are available for navigation error control through photogrammetric measurements or laser scanning during GNSS-outage, however, the overall processing complexity is still reserved for indirect estimation of system parameters, including the orientation errors and the sensor biases.

\subsection{INS/TAP System Integration}

The concept of rail-bound navigation integrates INS and track alignment positioning for maximising long-term performance, which aims at reducing the necessity of post-processing. To facilitate the real-time capability, a simplified approach is also introduced to lower the system complexity.

2.3.1 Body-to-track misalignment: Additional track orientation data, e.g. roll, pitch and heading, are directly available for orientation updates through the nominal misalignment between the system and the track as is shown by Equation (5). The misalignment between the track-level frame and IMU body frame is assumed to be constant, while the source of misalignment errors comprises track imperfections, harmonic vibrations and navigation errors.

$$
\tilde{C}_{b}^{n}=(I-\Psi) C_{t}^{n} C_{b}^{t}
$$

where $\tilde{C}_{b}^{n}$ is the INS-derived rotation from body to navigation frame; $C_{t}^{n}$ is the TAP-derived rotation of track in navigation frame; $C_{b}^{t}$ is the constant misalignment between body to track; $\Psi$ is the skew symmetric matrix for misalignment errors.

Despite the exactitude of track-derived orientation, the errors are apparent to be time-independent and practicable for navigation error control.

2.3.2 Processing alternative: The EKF and UKF are currently adopted for general POS to estimate indirect errors, e.g. orientation-related errors, to achieve long-term navigation performance. The orientation errors, however, are directly available from the track alignment positioning results with certain accuracy. In view of the steadiness of track alignment positioning, a simplified approach is introduced to remove the necessity of error propagation. The complementary filter is a possible alternative to Kalman or Particle filters to minimise the computational complexity (Neto et al., 2009), which is configured with low-pass and high-pass filters for data fusion:

$$
\hat{z}=G(s) z_{1}+[1-G(s)] z_{2}
$$

where $\hat{z}$ is the compensated output; $z_{1}$ and $z_{2}$ are two independent sources of output; $G(s)$ and $[1-G(s)]$ are the lowpass and high-pass filters respectively.

Although the complementary filter provides an alternative to simplify the data fusion of rail-bound navigation, the loss of 
statistical relationship across different parameters may introduce uncertain impacts into the entire system.

2.3.3 System implementation: Rail-bound navigation requires minimal input for initialisation (e.g. position or chainage) and maintains the navigation by inertial measurements and pre-defined track alignment data. Figure 2 illustrates the configuration of rail-bound navigation. The paths highlighted in green and blue correspond to the process of initialisation (and re-initialisation if necessary), and navigation error control respectively. Alternative input coloured red, such as external position or velocity data, is optional to the system for maximising the overall performance.

An initial chainage or position is given to the system for estimating the track-derived position and orientation, which provides the constant misalignment during initialisation process. After initialisation, rail-bound navigation is maintained by the inertial navigation system and controlled by position and orientation from TAP. The chainage is integrated from displacement or transformed from position, which connects the INS and TAP for continuous error control.

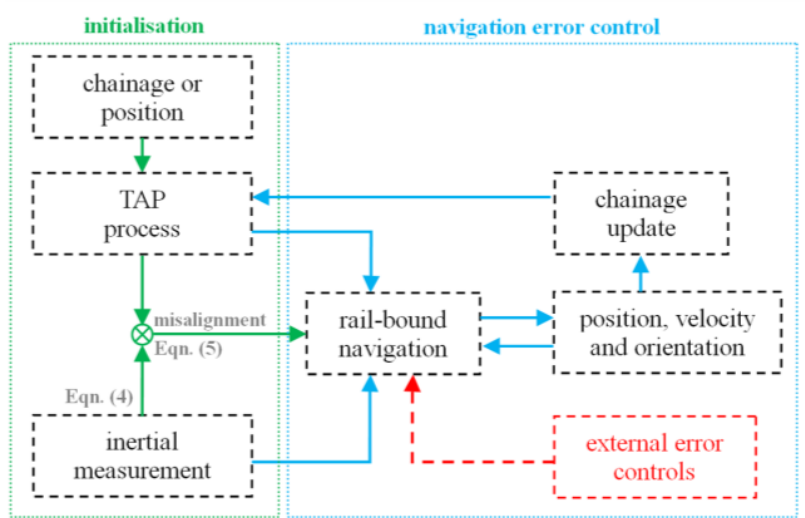

Figure 2. configuration of rail-bound navigation.

\section{EXPERIMENTAL STUDIES}

To examine the performance of the presented rail-bound navigation solution, an experiment was conducted for collecting inertial data via a small-scale railway model and motorised model train. The foci of the experiment are the overall performance in horizontal position, velocity and orientation.

\subsection{Hardware}

A consumer-grade MEMS IMU, freeIMU (Varesano, 2013), was employed for inertial data collection, which consists of a 6axis gyroscope and accelerometer (MPU6050) and a 3-axis magnetometer (HMC5883L). The freeIMU weighs about 2.5 grams and is only about $22 \mathrm{~mm}$ square. This IMU has been recently used for supporting orientation tracking and motion sensing, including autonomous aerial vehicle control (Choi et al., 2012; Liu and Prior, 2015) and gait analysis (Popescu and Miclea, 2015). An Arduino UNO Rev3 processing device and a Universal Serial Bus (USB) cable is utilised for interfacing the freeIMU to a notebook computer. The mounted device is shown in Figure 3.

The railway model is on a level surface and described by a track alignment. The alignment is compiled from the dimensions with arbitrary position and orientation. Figures 4 (A) and (B) show the railway model and the motorised model train respectively.

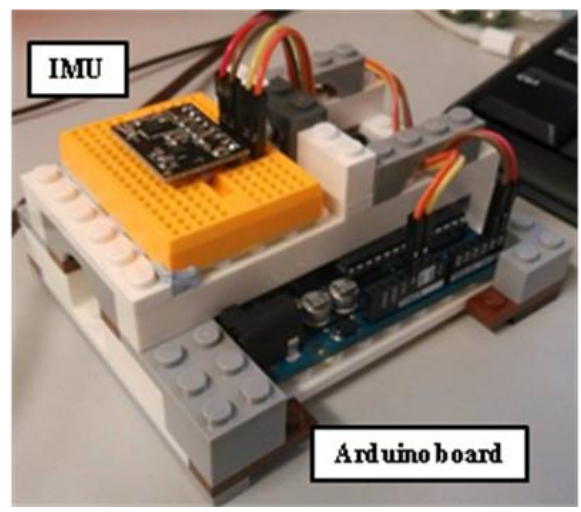

Figure 3. Mounted IMU device.

(A)

(B)
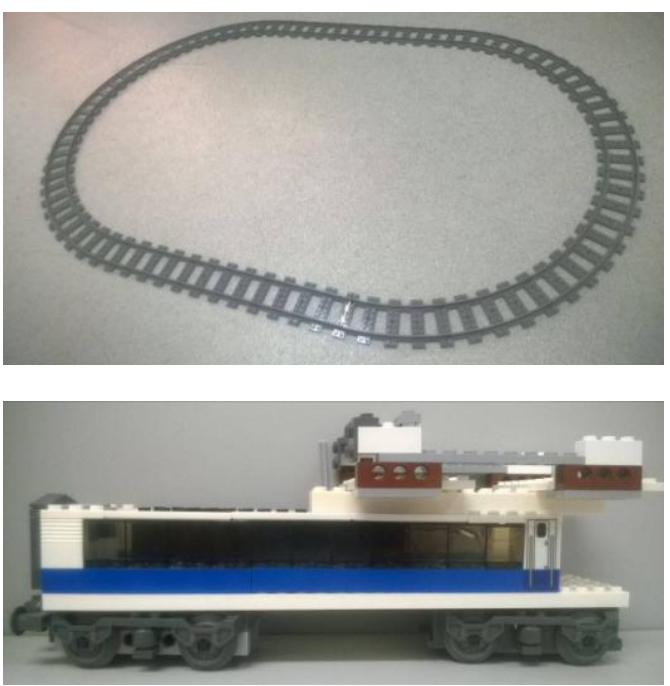

Figure 4. (A) Railway model. (B) Motorised model train.

Table 1 shows four sectional boundaries $(\mathrm{A}, \mathrm{B}, \mathrm{C}$ and $\mathrm{D})$ in Figure 4 (A) which correspond to the four tangent points of the model and are defined by chainage with heading and radius. The track position is referenced to $\mathrm{A}$ at $(10.000 \mathrm{~m}, 10.000 \mathrm{~m})$ and the track orientation is aligned with section A-B at $270.0^{\circ}$. A grid coordinate frame $(\mathrm{X}-\mathrm{Y}-\mathrm{Z})$ is defined by the track with the $\mathrm{X}-\mathrm{Y}$ plane parallel to horizontal plane and $\mathrm{Z}$ axis vertically pointing downward. In this experiment, the positioning results are fundamentally expressed in this frame by $\mathrm{X}, \mathrm{Y}$ and $\mathrm{H}$.

\begin{tabular}{|c|r|r|r|r|r|c|}
\hline & Chainage & Heading & Radius & \multicolumn{1}{c|}{ X } & \multicolumn{1}{c|}{ Y } & H \\
\hline A & 0.000 & $270.0^{\circ}$ & -- & 10.000 & 10.000 & 5.000 \\
B & 0.384 & $270.0^{\circ}$ & -0.320 & 10.000 & 9.616 & 5.000 \\
C & 1.388 & $90.0^{\circ}$ & -- & 9.361 & 9.616 & 5.000 \\
D & 1.772 & $90.0^{\circ}$ & -0.320 & 9.361 & 10.000 & 5.000 \\
\hline
\end{tabular}

Table 1. Track alignment of the railway model

\subsection{Experiment Configuration}

The railway model has dimensions of about $1.0 \mathrm{~m}$ by $0.7 \mathrm{~m}$, and consists of both straight and circular sections but not spirals, grades or cant. The track has a gauge of $42 \mathrm{~mm}$. 
Data collection comprised two loops of the track over a period of 15 seconds with simulated rail-bound motion. The train remained stationary for about 3 seconds during the initial and final phases, while the vehicle motion was manually controlled through switching on and off the power source.

Although the entire experiment is insufficient to represent longterm effects, the intention of experiment is to explore the capability and limitation of the rail-bound navigation solution.

\subsection{Data Processing}

For simplicity, the rail-bound navigation was implemented with complementary filters. The inertial measurements were collected at $120 \mathrm{~Hz}$, while the complementary filters were executed at $40 \mathrm{~Hz}$ to compensate the highly-dynamic motion.

Although the freeIMU is capable of inertial and magnetic field sensing, the magnetometer outputs were not used on account of the magnetic interference from the motor. In addition, the error controls for the sensor biases were not performed in this experiment on account of the simplified processing.

\subsection{Results and Analysis}

Two different processing scenarios were investigated, the pure inertial navigation and rail-bound navigation. The results are summarised and analysed in the following sections.

3.4.1 Inertial measurement data: The levelled accelerometer outputs and gyroscope outputs are presented in Figures 5 (A) and (B) respectively. The $\mathrm{x}, \mathrm{y}, \mathrm{z}$ outputs are coloured red, green and blue respectively. It is noted that the measurement noise of the accelerometer was considerably large, and the accelerations due to the train's motion cannot be clearly identified. In contrast, patterns can be observed from the sensed angular velocity in the body z-axis. The periodic chainage of the front axle is therefore recognisable.

A speed profile was generated by correlating the orientation pattern with track data through post-processing. The time of passing at the four sectional boundaries were recognised with travelling distance. Consequently, speed can be estimated and simulated the availability of non-drift external speed data for velocity control.

(A)
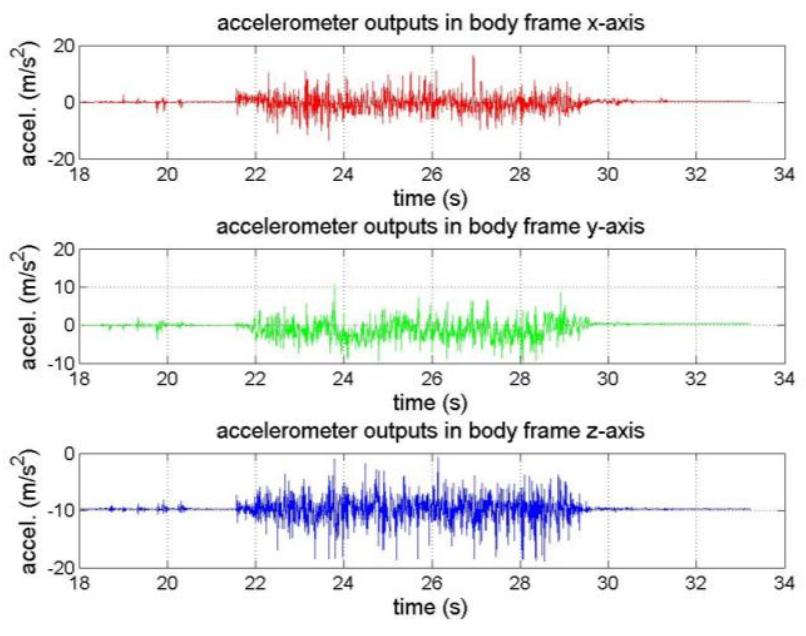

(B)
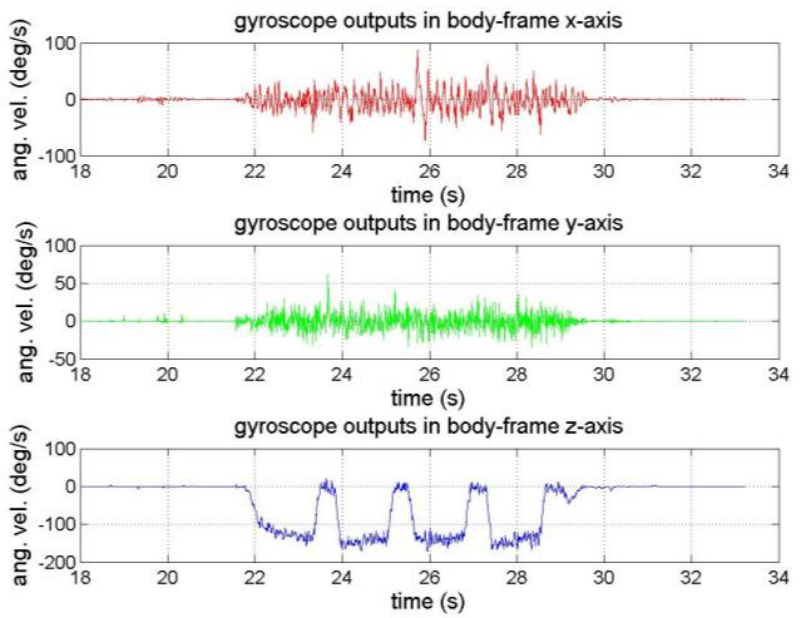

Figure 5. Body frame inertial measurement data, (A) Accelerometer outputs and (B) Gyroscope outputs.

3.4.2 Pure inertial navigation: The system is levelled by accelerometer outputs and aligned with track data during the initial stationary phase. In addition, a constant gravity is estimated from the accelerometer outputs to replace the global gravity model on account of localised mechanisation.

The INS-derived body-frame velocity and orientation are shown in Figure 6 (A) and (B) respectively. From the results, the velocity and orientation drifts are clearly observable. A linear growth of velocity drift for each body axis can be found in Figure 6 (B), resulting in the integrated velocity errors producing extreme position errors.

In Figure 7, the INS-derived horizontal position is mapped in red ( $1^{\text {st }}$ loop) and pink ( $2^{\text {nd }}$ loop) over the reference track, which illustrates critical positioning errors that the trajectory is completely incorrect.

The growth of velocity drift likely results either from inappropriate compensation of accelerometer bias, or inaccurate transformation and gravity compensation of the body acceleration. In light of the orientation drifts denoted in Figure 6 (B), the velocity drift is probably caused by the latter reason.

(A)
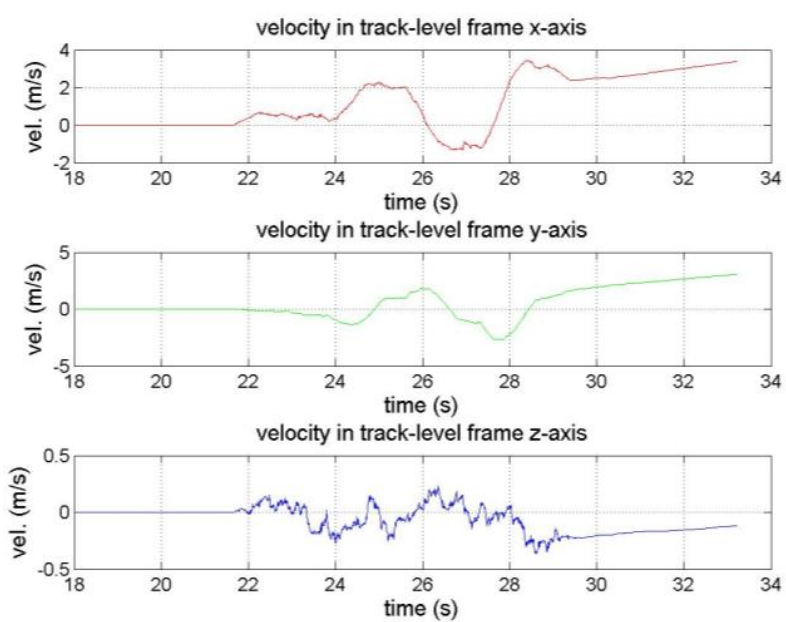
(B)
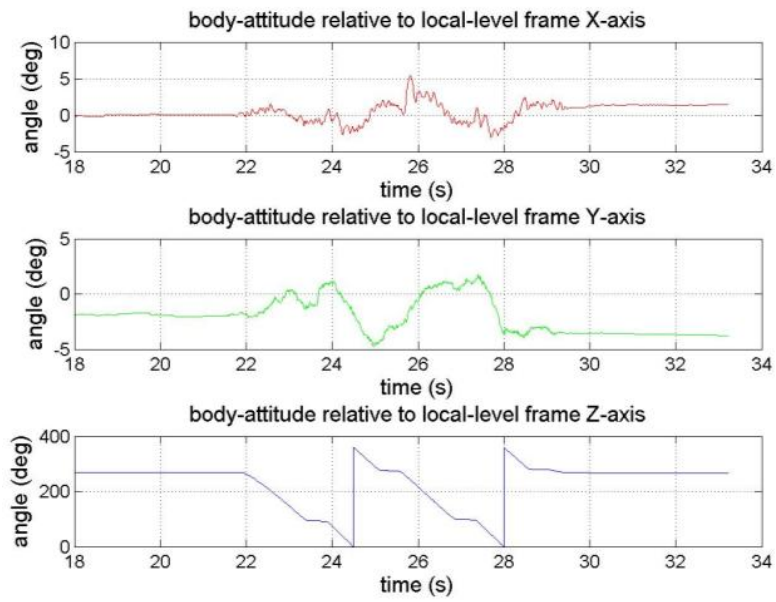

Figure 6. Pure inertial navigation results, (A) System velocity in body-frame and (B) System orientation.

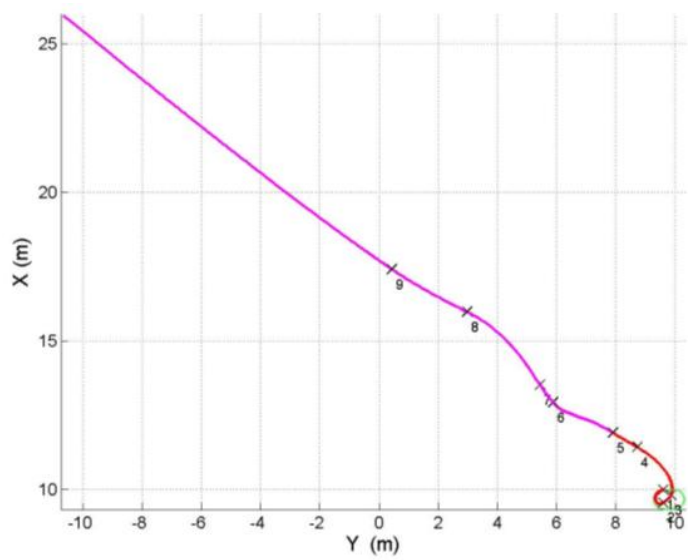

Figure 7. Pure inertial navigation position

A sequence of check point positions (1-9) are also indicated in Figure 7, which correspond to the reference boundaries (A, B, C and $\mathrm{D})$ of the track alignment traversed during the two loops. The position and orientation errors at the check points are expressed in track-level frame (x-y-z) and tabulated in Table 2. The orientation errors are expressed in roll-pitch-heading ( $\mathrm{r}-\mathrm{p}-$ h).

\begin{tabular}{|c|c|c|c|c|c|c|}
\hline \multirow{2}{*}{$\begin{array}{c}\text { Point } \\
\text { no. }\end{array}$} & \multicolumn{3}{|c|}{ Position error (m) } & \multicolumn{3}{|c|}{ Orientation error $\left({ }^{\circ}\right)$} \\
\hline & $\mathrm{x}$ & $\mathrm{y}$ & $\mathrm{z}$ & $\mathrm{r}$ & $\mathrm{p}$ & $\mathrm{h}$ \\
\hline 1 & 0.009 & 0.000 & 0.00 & 0.281 & -0.122 & -0.105 \\
\hline 2 & -0.002 & -0.210 & 0 & -1.313 & 0.660 & -0.230 \\
\hline 3 & -0.128 & & & & & 2.196 \\
\hline 4 & & & & & & 0.310 \\
\hline 5 & 88 & 5 & & 32 & -1 . & 4.913 \\
\hline 6 & -3.728 & & & & & 1.640 \\
\hline 7 & -4.554 & -4.1 & -0.0 & -0.939 & 2.769 & 7.406 \\
\hline 8 & 7.024 & & -0 . & 1.760 & -1.956 & 5.134 \\
\hline 9 & 9.179 & 7.423 & & 1.059 & -1.759 & -1.227 \\
\hline $\mathrm{M}$ & 4.381 & 3.762 & 0.152 & 1.176 & 2.060 & 3.567 \\
\hline
\end{tabular}

Table 2. Pure inertial navigation: position and orientation errors of check-points relative to the reference boundaries

According to Table 2, the RMS position errors in both $\mathrm{x}$ and $\mathrm{y}$ exceeded few meters; and that in $\mathrm{z}$ reached decimetre-level. The
RMS roll and pitch errors were about $1-2^{\circ}$. In contrast, the RMS heading error exceeded $3^{\circ}$. It is found that the heading errors accumulated and changed periodically, which may be caused by inaccurate compensation of heading when the train travelled across the sectional boundaries.

To summarise, the accumulation of orientation errors has significantly worsened the velocity estimation by incorrect transformation of acceleration vector and gravity compensation, which effectuated the excessive growth of position errors. The system orientation, as well as the gyroscope bias, should be properly controlled to achieve long-term performance.

3.4.3 Rail-bound navigation: Given the additional track data, the orientation and velocity drifts are extensively reduced through the rail-bound navigation implementation and the velocity update. The body-frame velocity and orientation through the rail-bound navigation are illustrated in Figures 8 (A) and (B) respectively. Through direct comparison, the velocity drifts were found to be minimised by the speed profile and appropriate orientation correction, while the impacts of gyroscope biases were notably reduced.

(A)
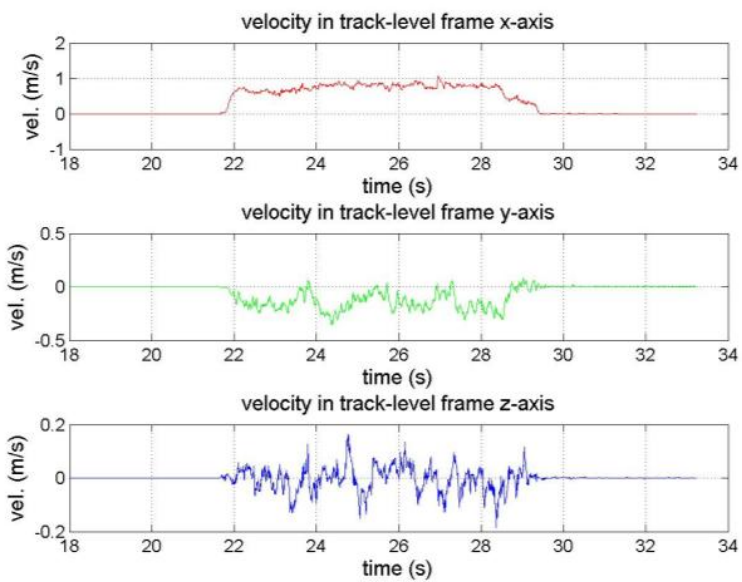

(B)
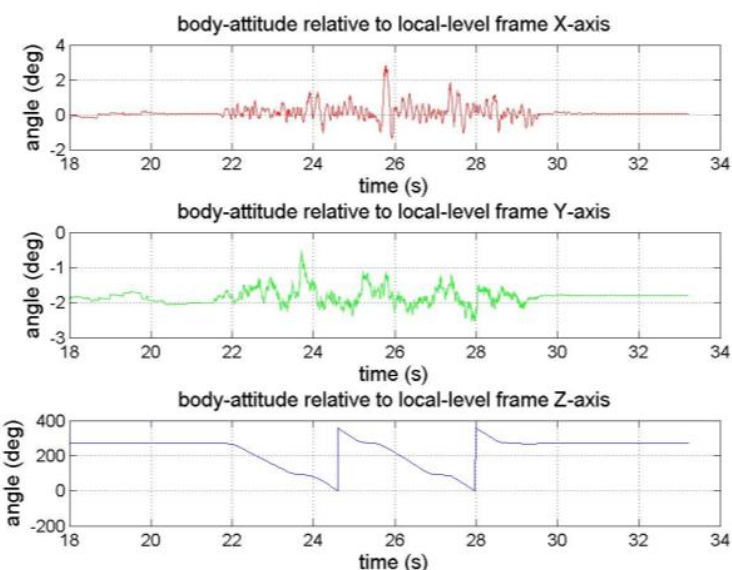

Figure 8. Rail-bound navigation results, (A) System velocity in body-frame and (B) System orientation.

Figure 9 illustrates that the rail-bound navigation provided a significant improvement in horizontal position accuracy over the pure inertial navigation results (Figure 7). Green lines 
represent the tracks; red and pink dots represent the computed positions in $1^{\text {st }}$ and $2^{\text {nd }}$ loops respectively. The position of four reference boundaries and the nine check points are also shown. Despite the local errors, Figure 9 shows that the overall trajectory fell within the track gauge thus reaching centimetrelevel accuracy in the lateral and down directions; however, the longitudinal position accuracy is relatively lower. The absolute position and orientation errors are expressed in track-level frame and presented in Table 3.

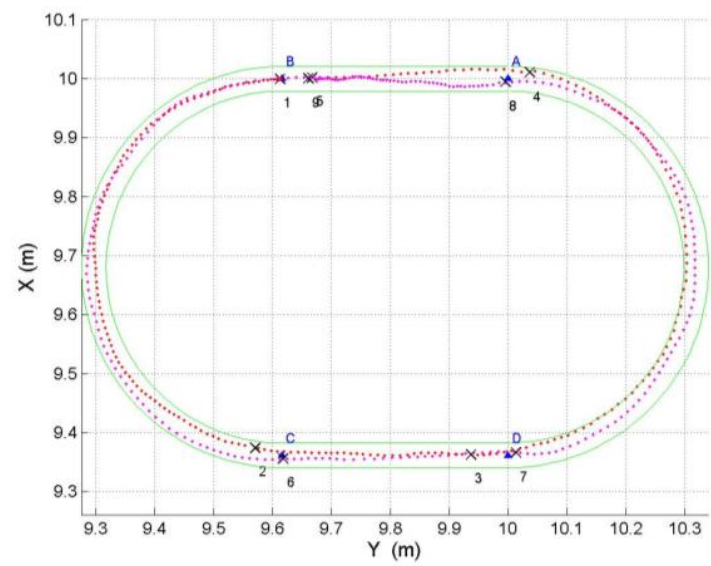

Figure 9. Rail-bound navigation position

\begin{tabular}{|c|r|r|r|r|r|r|}
\hline \multirow{2}{*}{$\begin{array}{c}\text { Point } \\
\text { no. }\end{array}$} & \multicolumn{2}{|c|}{ Position error $(\mathrm{m})$} & \multicolumn{3}{|c|}{ Orientation error $\left(^{\circ}\right)$} \\
\hline 1 & \multicolumn{1}{c|}{$\mathrm{x}$} & \multicolumn{1}{c|}{$\mathrm{y}$} & \multicolumn{1}{c|}{$\mathrm{z}$} & \multicolumn{1}{c|}{$\mathrm{r}$} & $\mathrm{p}$ & \multicolumn{1}{c|}{$\mathrm{h}$} \\
2 & -0.045 & -0.000 & 0.000 & -0.152 & -0.058 & -0.062 \\
3 & -0.063 & -0.001 & -0.001 & -0.390 & -0.342 & 7.394 \\
4 & -0.037 & 0.011 & 0.000 & 0.371 & 0.609 & 0.005 \\
5 & -0.051 & 0.002 & 0.000 & -0.105 & -0.157 & 6.884 \\
6 & 0.002 & 0.005 & 0.002 & -0.237 & -0.129 & 0.297 \\
7 & 0.014 & -0.005 & -0.006 & 0.204 & 0.014 & -0.004 \\
8 & 0.004 & -0.005 & -0.010 & 0.005 & -0.228 & -0.239 \\
9 & -0.045 & 0.000 & 0.001 & -0.084 & -0.092 & -1.722 \\
\hline RMS & 0.051 & 0.007 & 0.005 & 0.264 & 0.259 & 3.420 \\
\hline
\end{tabular}

Table 3. Rail-bound navigation: position and orientation errors of check-points relative to the reference boundaries

It can be seen from Table 3 that the RMS position errors in $y$ and $\mathrm{z}$ were few millimetres. In contrast, the RMS position error in $\mathrm{x}$ was $0.051 \mathrm{~m}$. Results indicated that the overall longitudinal errors were generally biased. One possible reason for the observed bias is the inaccuracy of the speed profile.

According to Table 3, the RMS roll and pitch errors were slightly larger than $0.2^{\circ}$, which is about $1 / 10^{\text {th }}$ of the pure inertial result; however, the RMS heading error remained at about $3.4^{\circ}$. It is noted that the dynamic misalignment was not modelled in this experiment. The train vibrations therefore contribute directly to the roll and pitch errors. In addition, the position errors in $\mathrm{x}$ introduce additional heading errors through inaccurate reference heading from TAP, which is worsened by the alignment's small radii of curvature. In this experiment, $0.02 \mathrm{~m}$ of position error in $\mathrm{x}$ can cause about $3.5^{\circ}$ of heading error at a radius of $0.32 \mathrm{~m}$. The heading errors are probably caused by the track geometry and position errors in $\mathrm{x}$.

From the rail-bound navigation results, the orientation errors are apparently reduced even though the system is inappropriately initialised and aligned. Consequently, the problem of velocity drift was controlled while the velocity accuracy was further improved by employing speed data.

\subsection{Experimental Restrictions}

The experimental results demonstrated a considerable improvement in position by applying the rail-bound navigation solution. Despite several limitations inherent in the simulated rail-bound system. The actual performance of the solution may not be fully represented by these results.

3.5.1 Nature of motion: Sudden acceleration and braking are resulted from a lack of fine motion control for the motorised model train. A rapid change of motion imparted by the on/off nature of the speed control and the lack of spirals between the straights and circular curves cannot be accurately measured by the freeIMU, which degrades the velocity estimation and requires additional speed data. A real-world train is usually subjected to steady acceleration and braking due to its mass and safety concerns, and the track alignments are substantially more sophisticated.

In addition, high frequency vibrations are clearly observable in Figures 5 (A) and (B), which dominated the actual motion and degraded the navigation performance. For real-world train systems such as those operated by Hong Kong's Mass Transit Railway Corporation (MTRC), high frequency vibrations are usually absorbed by the bogies and suspension systems, which would provide a more favourable environment for inertial measurements. Consequently, rail-bound navigation is expected to provide more reliable results under real-world conditions.

3.5.2 Track design: The model track only consisted of straight and circular sections, which is an over-simplification. In reality, the introduction of spiral curve sections, changes in cant and grade, and physical track defects are some potential impacts on real-world navigation performance. Consequently, field studies aboard operating trains are required to assess and refine the rail-bound navigation solution.

3.5.3 Model scale: A critical issue for the validity of these experimental results is the model scale, including track size, period of motion sensing and motion dynamics. Although the experiment is not designed to investigate the long-term effects of the proposed solution, the importance of real-world operation should not be ignored.

\section{CONCLUSIONS AND FURTHER STUDIES}

This paper has suggested an alternative approach, rail-bound navigation, for replacing the GNSS component in a POS in railway systems. The proposed method is potentially applicable to underground railway systems for navigation and direct georeferencing.

An experiment was conducted with a small-scale railway model to examine the performance and limitations of the rail-bound navigation system. Despite the system model's simplicity, an overall improvement, including position, velocity and orientation, has been illustrated in the results by the application of track alignment data.

Based on a preliminary analysis of data acquired on board one of Hong Kong's MTRC trains, rail-bound navigation is capable of maintaining decimetre to metre-level accuracy in the longitudinal direction for a section over $1000 \mathrm{~m}$ in length 
without external speed data and post-processing. To ascertain the performance of rail-bound navigation, further studies should be conducted for the development of rigorous solution; the enhancement of performance stability; and the quantitative analysis for navigation accuracy.

\section{REFERENCES}

Choi, M. H., Porter, R., and Shirinzadeh, B., 2013. Comparison of attitude determination methodologies with low cost inertial measurement unit for autonomous aerial vehicle. In: 2013 IEEE/ASME International Conference on Advanced Intelligent Mechatronics, pp. 1349-1354.

Chow, R., 2011. Evaluating inertial measurement units. EDN Network.

Ellum, C., and El-Sheimy, N., 2002. Land-based mobile mapping systems. Photogrammetric Engineering \& Remote Sensing, 68(1), pp. 13, 15-17, 28.

El-Sheimy, N., 2005. An Overview of Mobile Mapping Systems. In: FIG Working Week 2005 and GSDI 8, Cairo, Egypt, 26 pages.

Farrell, J. A., 2008. Aided Navigation: GPS with High Rate Sensors. McGraw-Hill.

Gräfe, G., 2007. Quality management in kinematic laser scanning applications. In: The International Archives of Photogrammetry, Remote Sensing and Spatial Information Sciences, Padova, Italy, Vol. XXXVI, Part 5/C55, 6 pages.

Gräfe, G., 2008. Kinematic 3D laser scanning for road or railway construction surveys. In: Proceedings of the International Conference on Machine Control \& Guidance, pp. 24-26.

Grewal, M. S., Weill, L. R., and Andrews, A. P., 2007. Global Positioning Systems, Inertial Navigation, and Integration. John Wiley \& Sons, Inc.

Hassan, T., Ellum, C., and El-Sheimy, N., 2006. Bridging landbased mobile mapping using photogrammetric adjustments. In: Proceedings of the 2006 ISPRS Commission I Symposium, From Sensors to Imagery, Paris, France, pp. 128-139.

Hung, R., King, B., and Chen, W., 2015. Conceptual issues regarding the development of underground railway laser scanning systems. ISPRS Int. J. Geo-Inf, 4, pp. 185-198.

Imanishi, A., Tachibana, K., and Tsukahara, K., 2011. The development of accuracy maintenance method for mobile mapping system (MMS) data at GPS invisible area. In: Proceedings of the FIG Working Week 2011, Marrakech, Morocco (18-22 May 2011).

Klein, I., and Filin, S., 2011. LiDAR and INS fusion in periods of GPS outages for mobile laser scanning mapping systems. Int. Arch. Photogramm. Remote Sens. Spat. Inf. Sci., Vol. XXXVIII, Part 5/W12, pp. 231-236.

Leslar, M., Perry, G., and McNease, K., 2010. Using mobile lidar to survey a railway line for asset inventory. ASPRS 2010 Annual Conference, San Diego, CA, USA (26-30 April 2010).
Liu, C., and Prior, S., 2015. Design and implementation of a mini quadrotor control system in GPS denied environments. In: 2015 International Conference on Innovation, Communication and Engineering, Xiangtan, CN (23-28 October 2015).

Nassar, S., Shin, E., Niu, X., and El-Sheimy, N., 2005. Accurate INS/GPS positioning with different inertial systems using various algorithms for bridging GPS outages. In: Proceedings of the 18th International Technical Meeting of the Satellite Division of The Institute of Navigation, Long Beach, CA, USA (13-16 September 2005).

Neto, A., Macharet, D., Campos V., Campos M., 2009. Adaptive complementary filtering algorithm for mobile robot localization. Journal of the Brazilian Computer Society, 15(3), pp. 19-31.

Popescu, A. T., and Miclea, L., 2015. Design and prototype of a gait analysis device. Acta Electrotehnica, 56(3), pp. 276-279.

Puente, I., González-Jorge, H., Arias, P., and Armesto, J., 2011. Land-based mobile laser scanning systems: a review. In: The International Archives of the Photogrammetry, Remote Sensing and Spatial Information Sciences, Vol. XXXVIII, Part 5/W12, pp. 163-168.

Puente, I., González-Jorge, H., Martínez-Sánchez, J., and Arias, P., 2013. Review of mobile mapping and surveying technologies. Measurement, 46(7), pp. 2127-2145.

Rogers, R. M., 2000. Applied Mathematics in Integrated Navigation Systems. Reston, VA: American Institute of Aeronautics and Astronautics.

Varesano, F., 2013. FreeIMU: An Open Hardware Framework for Orientation and Motion Sensing.

Woodman, O.J., 2007. An Introduction to Inertial Navigation. University of Cambridge. 